\title{
YpT1-2NO rectal cancer after neoadjuvant chemoradiation has lower survival compared with pT1-2N0 rectal cancer
}

\author{
Jue-feng Wan ${ }^{1,2}$, Ji Zhu ${ }^{1,2}$, Gui-chao Li ${ }^{1,2}$, Wen-jie Sun ${ }^{1,2}$, Zhen Zhang ${ }^{1,2}$ \\ ${ }^{1}$ Department of Radiation Oncology, Fudan University Shanghai Cancer Center, Fudan University, Shanghai, China \\ ${ }^{2}$ Department of Oncology, Shanghai Medical College, Fudan University, Shanghai, China \\ Correspondence to: \\ Zhen Zhang, e-mail: zhenzhang6@hotmail.com \\ Keywords: rectal cancer, adjuvant chemotherapy, risk factors, good response, SEER \\ Received: July 09, $2015 \quad$ Accepted: September 02, 2015 Published: October 21, 2015
}

\section{ABSTRACT}

\begin{abstract}
Pathologic T1-2NO rectal cancer shows an excellent prognosis without preoperative or postoperative chemoradiation. However, oncologic outcome of ypT1-2NO remains unclear and undetermined. Thus, the aim of this study was to compare the survival of ypT1-2 and pT1-2 rectal cancer patients after radical resection and identify risk factors of ypT1-2 rectal cancer in Surveillance, Epidemiology, and End Results Program (SEER)-registered rectal cancer patients. The results showed that ypT1-2NO rectal cancer after neoadjuvant chemoradiation has lower survival compared with pT1-2NO rectal cancer and mucinous/signet-ring cancer and less than 12 lymph nodes retrieval were two risk factors in ypT1-2 patients. These results suggest that ypT1-2 patients with one or two risk factors may benefit from postoperative adjuvant chemotherapy.
\end{abstract}

\section{INTRODUCTION}

Colorectal cancer is one of the most common cancers in the western world and preoperative chemoradiotherapy (CRT) followed by total mesorectal excision is the standard of care for patients with locally advanced rectal cancer [1-5]. Park et al. revealed that treatment response to neoadjuvant chemoradiotherapy was an early surrogate marker and tumor response was associated with 5-year recurrence free survival [6]. Although it is well known that complete pathologic response to chemoradiation is associated with excellent prognosis, there are few studies evaluating the oncologic outcome of patients with ypT1-2N0 rectal cancer who underwent preoperative chemoradiation.

Actually, pathologic T1-2N0 rectal cancer shows an excellent prognosis without preoperative or postoperative chemoradiation. However, oncologic outcome of ypT1-2N0 remains unclear and undetermined. Thus, the aim of this study was to compare the cancer specific survival of ypT1-2 and pT1-2 rectal cancer patients after radical resection and identify risk factors of ypT1-2 rectal cancer.

\section{RESULTS}

\section{Patient characteristics}

We identified 10,673 eligible elderly patients in SEER database during the 9-year study period (between 2004 and 2012), which included 8,433 patients in pT1-2 and 2,240 patients in ypT1-2. There were 6167 $(57.8 \%)$ males and $4506(42.2 \%)$ females. Patient demographics and pathological features are summarized in Table 1.

Clinicopathological differences between the two groups

When compared among two subgroups, it was investigated that significant differences were found among the sex (more female in pT1-2, $p<0.001$ ), race, pathological grading, histological type (more mucinous / signet ring cell in ypT1-2, $p<0.001$ ), stage (more pT1 in pT1-2, $p<0.001$ ) and current standard (more cases with $\geq 12$ LNs dissected in ypT1-2, $p<0.001$ ). (Table 1 ). 
Table 1: Patient characteristics

\begin{tabular}{|c|c|c|c|c|}
\hline \multirow[b]{2}{*}{ Variable } & Total & pT1-2 & урТ1-2 & \multirow[b]{2}{*}{$P$ value } \\
\hline & $n=10,673$ & $n=8,433$ & $n=2,240$ & \\
\hline Sex & & & & $<0.001$ \\
\hline Male & 6167 & $55.9 \%$ & $64.9 \%$ & \\
\hline Female & 4506 & $44.1 \%$ & $35.1 \%$ & \\
\hline Race & & & & $<0.001$ \\
\hline White & 8836 & $83.1 \%$ & $81.6 \%$ & \\
\hline Black & 796 & $6.9 \%$ & $9.5 \%$ & \\
\hline Other & 1041 & $10 \%$ & $8.9 \%$ & \\
\hline Pathological grading & & & & 0.048 \\
\hline High/Moderate & 8603 & $81 \%$ & $79 \%$ & \\
\hline Poor/undifferentiation & 804 & $7.3 \%$ & $8.6 \%$ & \\
\hline Unknown & 1266 & $11.7 \%$ & $12.4 \%$ & \\
\hline Histological type & & & & $<0.001$ \\
\hline Adenocarcinoma & 10465 & $98.4 \%$ & $97 \%$ & \\
\hline Mucinous /Signet ring cell & 208 & $1.6 \%$ & $3 \%$ & \\
\hline Stage & & & & $<0.001$ \\
\hline pT1+ypT1 & 6509 & $66.9 \%$ & $38.3 \%$ & \\
\hline pT2+ypT2 & 4164 & $33.1 \%$ & $61.2 \%$ & \\
\hline No. of LNs dissected & & & & $<0.001$ \\
\hline$<12$ & 7013 & $66.6 \%$ & $62.2 \%$ & \\
\hline$\geq 12$ & 3660 & $33.4 \%$ & $37.8 \%$ & \\
\hline
\end{tabular}

\section{Cancer specific survival between the two groups}

The 5-year CSS was $92.2 \%$ in pT1-2 and $87.5 \%$ in ypT1-2 and the 5-year overall survival was $84 \%$ in pT1-2 and $80.6 \%$ in ypT1-2, which had significant difference in univariate log-rank test $(P<0.001$ and $P=0.003$, respectively) (Fig. 1 and 2). Besides, black race $(P<0.001)$, poor or undifferentiation tumor grade $(P=0.002)$, mucinous/signet-ring cancer $(P<0.001)$, pT2 $(P<0.001)$, and less number in LNs dissection $(p=0.001)$ were identified as significant risk factors for poor survival on univariate analysis (Table 2). When multivariate analysis with Cox regression was performed, we convinced the above five factors also as independent prognostic factors (Table 2).

\section{Potential risk factors and prognostic significance in ypT1-2}

All potential risk factors, including gender, race, pathological grading, histological type, stage and No. of LNs dissected were evaluated by using the KaplanMeier method (compared with Log rank test). Among these potential risk factors, race, histological type and
No. of LNs dissected exhibited a correlation with CSS (Table 3). Cox multivariate regression analysis revealed only two factors to be associated with CSS: histological type and No. of LNs dissected (Table 3). The 5-year CSS in patients with none, one or two risk factors was $90.5 \%$, $86.5 \%$ and $65.6 \%$, respectively $(p<0.001)$ (Fig. 3 ).

\section{DISCUSSION}

Two retrospective studies investigated the oncologic outcomes in patients with ypT1-2N0 rectal cancer who underwent CRT and radical surgery and compare with those who did not receive preoperative CRT [10-11]. They found similar results that no significant difference was observed in the 5-year local recurrence rate and overall survival for two groups. However, the number of patients in these two studies is too small to provide adequate power for drawing any definitive conclusions regarding oncologic outcomes.

In the present study, we identified 8,433 patients in pT1-2 and 2,240 patients in ypT1-2. The 5-year CSS was $92.2 \%$ in pT1-2 and $87.5 \%$ in ypT1-2 $(P<0.001)$. Thus, ypT1-2N0 rectal cancer after neoadjuvant chemoradiation has lower survival compared with pT1-2N0 rectal 


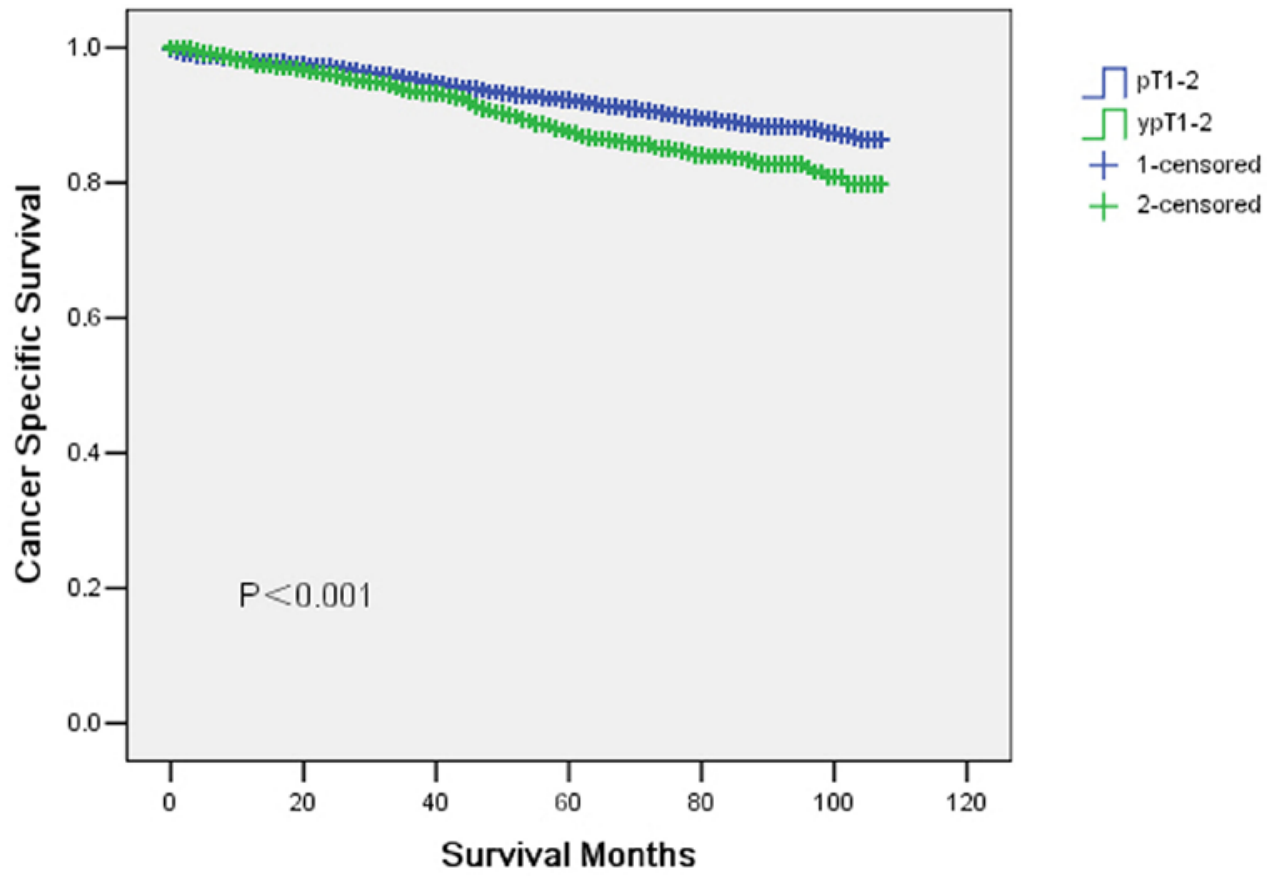

Figure 1: Cancer specific survival curves in yT1-2 and ypT1-2 rectal cancer patients. The 5-year cancer specific survival (CSS) was $92.2 \%$ in pT1-2 and $87.5 \%$ in ypT1-2 $(p<0.001)$.

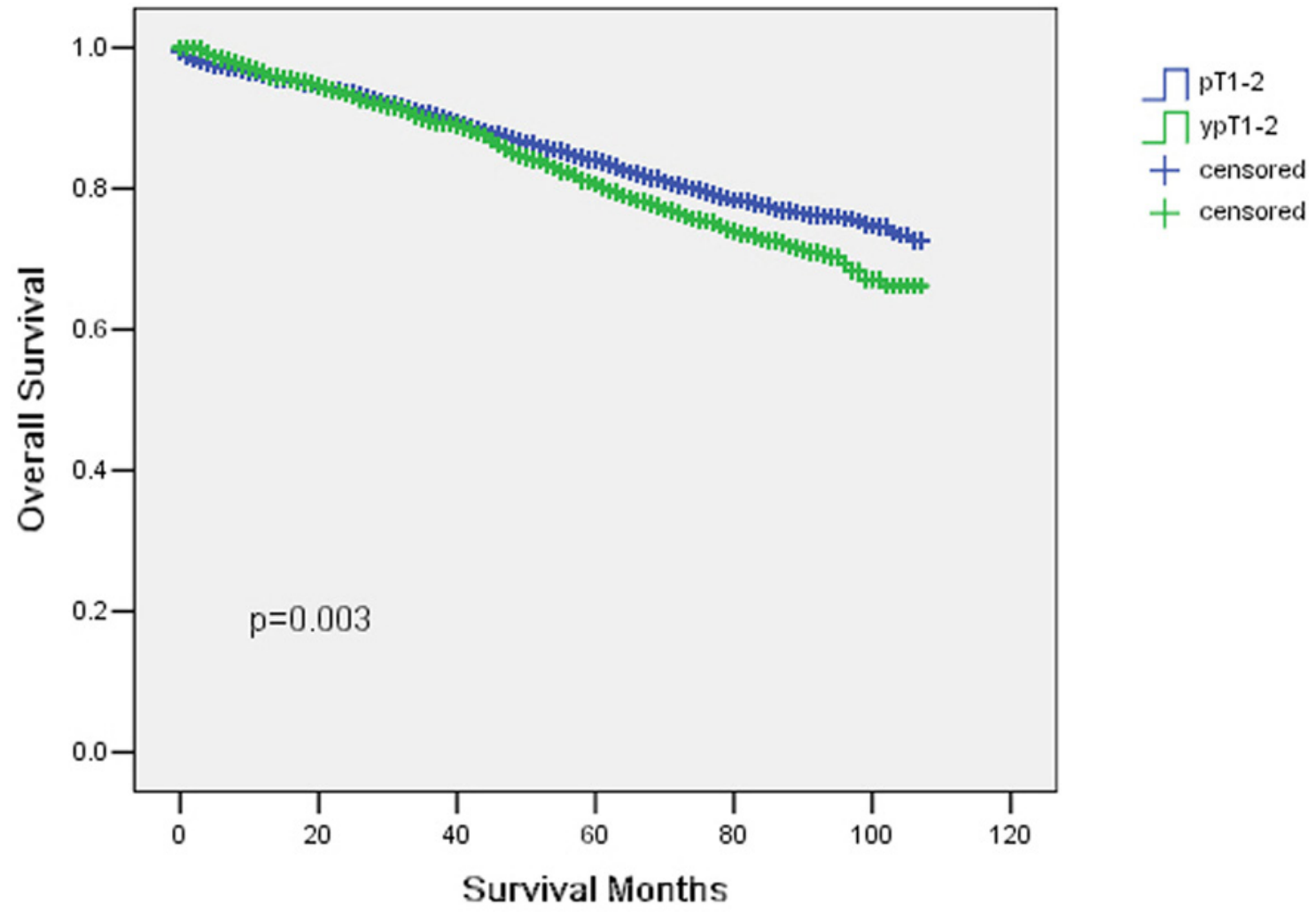

Figure 2: Overall survival curves in yT1-2 and ypT1-2 rectal cancer patients. The 5 -year overall survival (OS) was $84 \%$ in pT1-2 and $80.6 \%$ in ypT1-2 $(p=0.003)$. 
Table 2: Univariate and multivariate survival analyses of rectal cancer patients according to various clinicopathological variables

\begin{tabular}{|c|c|c|c|c|}
\hline Variable & $n$ & $\begin{array}{c}\text { 5-year CSS } \\
(\%)\end{array}$ & Univariate $P$ & Multivariate $\mathrm{P}$ \\
\hline Sex & & & 0.899 & 0.938 \\
\hline Male & 6167 & 91 & & \\
\hline Female & 4506 & 91.4 & & \\
\hline Race & & & $<0.001$ & $<0.001$ \\
\hline White & 8836 & 91.1 & & \\
\hline Black & 796 & 87.5 & & \\
\hline Other & 1041 & 94 & & \\
\hline Pathological grading & & & 0.002 & 0.015 \\
\hline High/Moderate & 8603 & 91.4 & & \\
\hline Poor/undifferentiation & 804 & 86.9 & & \\
\hline Unknown & 1266 & 92.3 & & \\
\hline Histological type & & & $<0.001$ & 0.007 \\
\hline Adenocarcinoma & 10465 & 91.4 & & \\
\hline Mucinous /Signet ring cell & 208 & 81.8 & & \\
\hline Stage & & & $<0.001$ & $<0.001$ \\
\hline pT1+ypT1 & 6509 & 93.3 & & \\
\hline pT2+ypT2 & 4164 & 87.9 & & \\
\hline No. of LNs dissected & & & 0.001 & $<0.001$ \\
\hline$<12$ & 7013 & 90.6 & & \\
\hline$\geq 12$ & 3660 & 92.3 & & \\
\hline Stage & & & $<0.001$ & 0.004 \\
\hline pT1-2 & 8433 & $92.2 \%$ & & \\
\hline урТ1-2 & 2240 & $87.5 \%$ & & \\
\hline
\end{tabular}

cancer. The standard treatment for T1-2N0 disease is surgery alone without preoperative or postoperative CRT. Actually, pathologic T1-2N0 rectal cancer shows an excellent prognosis without postoperative chemoradiation. In contrast, clinical practice guideline of adjuvant chemotherapy of ypT1-2 rectal cancer is not based on solid evidence and the level of scientific evidence for sufficient benefit is much lower than colon cancer [12-15].

The recently reported meta-analysis of 21 RCTs showed that a significant reduction in the risk of death $(17 \%)$ and in the risk of disease recurrence $(25 \%)$ among patients with rectal cancer undergoing adjuvant chemotherapy as compared to those undergoing observation [16]. However, only one of these 21 RCTs contained patients treated with preoperative chemoradiotherapy and almost all of these patients underwent curative resection of rectal cancer without preoperative treatment. However, things are more complicated in the era of the wide use of neoadjuvant chemoradiation for patients with locally advanced rectal cancer.

The European Organization for Research and Treatment of Cancer (EORTC) 22921 trial did not confirm a significant disease-free or overall survival benefit for adjuvant FU-based chemotherapy for locally advanced rectal cancer [17]. A second analysis of the EORTC 22921 trial was performed to find whether there is a subset of patients who, after preoperative radiotherapy or chemoradiotherapy and surgery, may benefit from adjuvant postoperative FU/leucovorin chemotherapy. Exploratory analyses suggest that only good-prognosis patients (ypT0-2) benefit from adjuvant chemotherapy [18].

However, two retrospective studies did not find patients with ypT1-2 benefit from postoperative adjuvant chemotherapy [19-20]. Fietkau et al. showed that 3-year disease free survival (DFS) for patients without lymph 
Table 3: Univariate and multivariate survival analyses of ypT1-2 rectal cancer patients according to various clinicopathological variables

Variable
\begin{tabular}{|l|c|c|c|c|}
\hline Sex & & & 0.75 & 0.615 \\
\hline Male & 1453 & 86.6 & & \\
\hline Female & 787 & 89.2 & & \\
\hline Race & & & 0.009 & 0.352 \\
\hline White & 1828 & 87.6 & & \\
\hline Black & 211 & 81 & & \\
\hline Other & 201 & 93.6 & & \\
\hline Pathological grading & & & 0.052 & 0.861 \\
\hline High/Moderate & 1769 & 88.3 & & \\
\hline Poor/undifferentiation & 193 & 79.9 & & \\
\hline Unknown & 278 & 88 & & $<0.001$ \\
\hline Histological type & & & & \\
\hline Adenocarcinoma & 2171 & 88 & & \\
\hline Mucinous/Signet ring cell & 69 & 71.2 & & \\
\hline Stage & & & & \\
\hline ypT1 & 870 & 87.5 & & 0.001 \\
\hline ypT2 & 1370 & 87.4 & & \\
\hline No. of LNs dissected & & & & \\
\hline$<12$ & 1394 & 85.9 & & \\
\hline$\geq 12$ & & 90.3 & & \\
\hline
\end{tabular}

node metastases (ypN0) was excellent, independent of whether they had received postoperative chemotherapy $(87.5 \pm 6.0$ percent $)$ or not $(87.7 \pm 6.7$ percent $)$. In addition, SEER-Medicare-linked database showed that patients who had already received 5-FU-based neoadjuvant chemoradiotherapy, postoperative 5-FU-based chemotherapy did not prolong cancer-specific survival (CSS) in ypT1-2 $(P=0.960)$.

Thus, Up to date, no general agreement has been reached on the indications of adjuvant chemotherapy for ypT1-2 patients. In our study, we identified histological type and No. of LNs dissected as two significant risk factors for survival on univariate and multivariate analysis. Thus, ypT1-2 patients with one or two risk factors may benefit from postoperative adjuvant chemotherapy.

Although this is a large population-based study, it has several potential limitations. First, the SEER database lacks several important tumor characteristics (eg, perineural invasion, lymphovascular invasion and distance from anal verge). Thus, our analyses could not adjust for these potential confounding factors. Second, our study is the lack of data in the SEER registry on the use of chemotherapy, resulting in a potentially significant confounder in the current study. It is possible that patients may have received adjuvant chemotherapy. Still, our study has its convincing power for its larger population based study.

In conclusion, ypT1-2N0 rectal cancer after neoadjuvant chemoradiation has lower survival compared with pT1-2N0 rectal cancer and ypT1-2 patients with one or two risk factors may benefit from postoperative adjuvant chemotherapy.

\section{MATERIALS AND METHODS}

\section{Patient selection in the SEER database}

The SEER, a population-based reporting system, was surveyed for the retrospective collection of data used in the analysis. The SEER program collects and publishes cancer incidence and survival data from 18 populationbased cancer registries, covering $>25 \%$ of the US population. Because no personal identifying information was used in the analysis, this study was granted an 


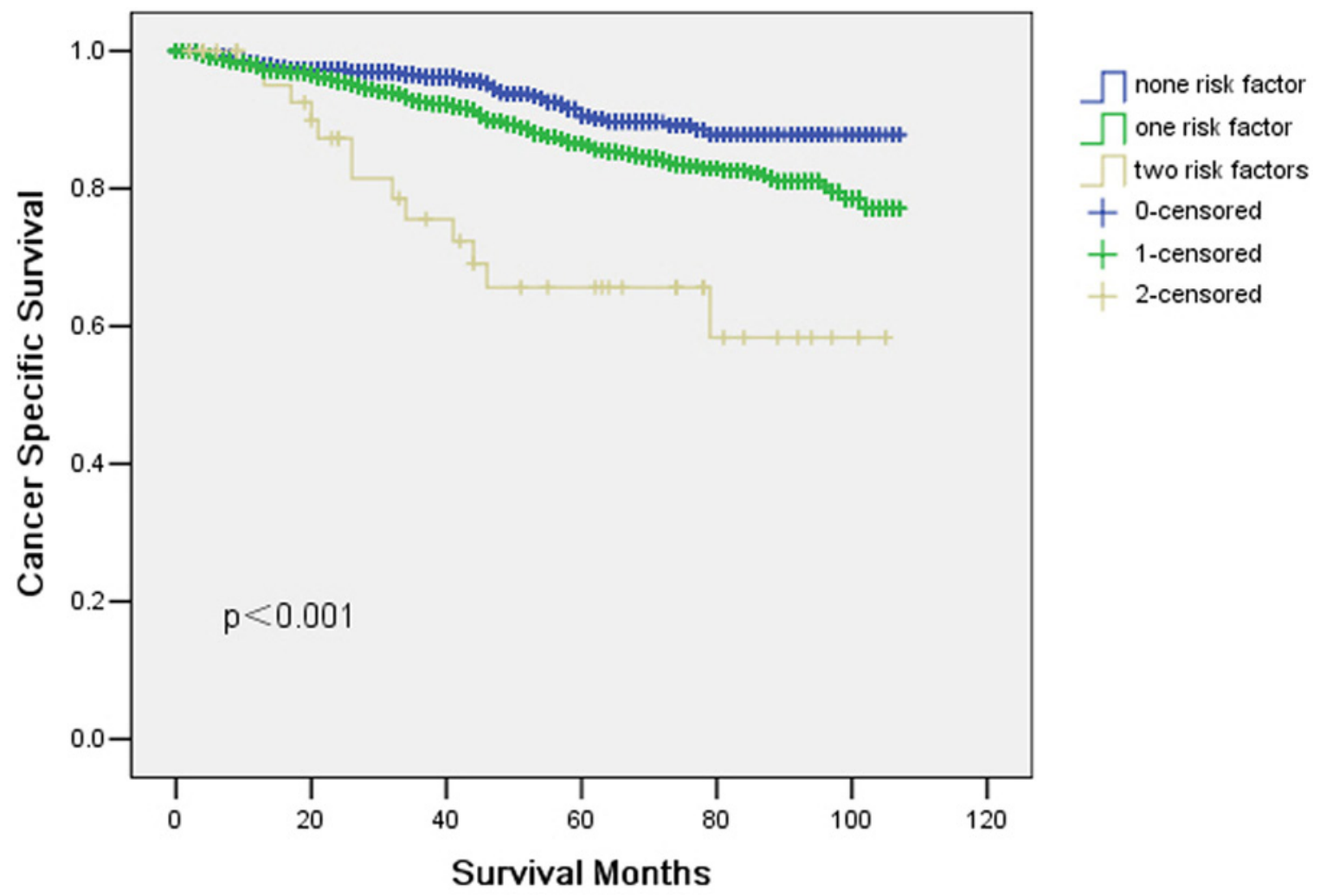

Figure 3: Cancer specific survival (CSS) in ypT1-2 rectal cancer patients according to number of risk factors. The 5 -year cancer specific survival (CSS) in patients with none, one or two risk factors was $90.5 \%, 86.5 \%$ and $65.6 \%$, respectively $(p<0.001)$.

exemption from the Institutional Review Board of the study institution on March 30, 2012.

Cases of rectal cancer (C20.9 Rectum, NOS) from 2004 to 2012 were extracted from the SEER database (SEER*Stat 8.2.1) according to the Site Recode classifications with limitation to radiation prior to surgery and radiation preoperatively and post-surgery. Histological type were limited to adenocarcinoma (ICD-03, 8140/3, 8210/3, $8261 / 3$, 8263/3), mucinous adenocarcinoma (ICD-03, 8480/3), and signet ring cell carcinoma (ICD-03, 8490/3). We selected this range because American Joint Committee on Cancer (AJCC) TMN stage was available since 2004 and chemoradiation has become the standard treatment since the landmark German CAO/ARO/AIO-94 trial using preoperative chemoradiation which was published in 2004. Other exclusion criteria were as follows: more than one primary cancer but the rectal cancer wasn't the first one, synchronous distance metastases, and patients with unknown TNM stage.

\section{Statistical analysis}

Gender, race, pathological grading, histological type, stage, No. of lymph nodes (LNs) dissected and cancer specific survival (CSS) were extracted from SEER database. CSS was calculated from the date of diagnosis to the date of cancer specific death. Deaths attributed to the rectal cancer were treated as events and deaths from other causes were treated as censored observations. The Kaplan-Meier method was used to estimate the CSS [7]. The association between each of the potential prognostic factors and the estimated CSS was tested with the log-rank test [8]. Multivariate analysis was performed using the Cox regression model [9]. The statistical test was two sided and $P<0.05$ was considered statistically significant. PASW Statistics 13 (SPSS Inc., Chicago, USA) was used for the statistical analysis.

\section{ACKNOWLEDGMENTS}

The authors acknowledge the efforts of the Surveillance, Epidemiology, and End Results (SEER) Program tumor registries in the creation of the SEER database. The interpretation and reporting of these data are the sole responsibility of the authors. The authors have no support or funding to report.

\section{Authors' contributions}

JFW and ZZ conceived of and designed the study. JFW, JZ, GCL and WJS performed the analyses. JZ and GCL prepared all tables. JFW and ZZ wrote the main manuscript. All authors reviewed the manuscript. 


\section{CONFLICTS OF INTEREST}

The authors declare that they have no competing interests.

\section{REFERENCES}

1. Aschele C, Cionini L, Lonardi S, Pinto C, Cordio S, Rosati G, Artale S, Tagliagambe A, Ambrosini G, Rosetti P, Bonetti A, Negru ME, Tronconi MC, Luppi G, Silvano G, Corsi DC, et al. Primary tumor response to preoperative chemoradiation with or without oxaliplatin in locally advanced rectal cancer: pathologic results of the STAR-01 randomized phase III trial. J Clin Oncol. 2011; 29:2773-2780.

2. Gerard JP, Azria D, Gourgou-Bourgade S, Martel-Laffay I, Hennequin C, Etienne PL, Vendrely V, Francois E, de La Roche G, Bouche O, Mirabel X, Denis B, Mineur L, Berdah JF, Mahe MA, Becouarn Y, et al. Comparison of two neoadjuvant chemoradiotherapy regimens for locally advanced rectal cancer: results of the phase III trial ACCORD 12/0405-Prodige 2. J Clin Oncol. 2010; 28:1638-1644.

3. Rodel C, Liersch T, Becker H, Fietkau R, Hohenberger W, Hothorn T, Graeven U, Arnold D, Lang-Welzenbach M, Raab HR, Sulberg H, Wittekind C, Potapov S, Staib L, Hess C, Weigang-Kohler K, et al. Preoperative chemoradiotherapy and postoperative chemotherapy with fluorouracil and oxaliplatin versus fluorouracil alone in locally advanced rectal cancer: initial results of the German CAO/ARO/AIO-04 randomised phase 3 trial. Lancet Oncol. 2012; 13:679-687.

4. Bisceglia G, Mastrodonato N, Tardio B, Mazzoccoli G, Corsa P, Troiano M, Parisi S. Intermediate neoadjuvant radiotherapy for T3 low/middle rectal cancer: post-operative outcomes of a non-controlled clinical trial. Oncotarget. 2014; 5:11143-53.

5. Napolitano M, D'Alterio C, Cardone E, Trotta AM, Pecori B, Rega D, Pace U, Scala D, Scognamiglio G, Tatangelo F, Cacciapuoti C, Pacelli R, Delrio P. Scala. Peripheral myeloid-derived suppressor and $\mathrm{T}$ regulatory PD-1 positive cells predict response to neoadjuvant shortcourse radiotherapy in rectal cancer patients. Oncotarget. 2015; 6:8261-70.

6. Park IJ, You YN, Agarwal A, Skibber JM, RodriguezBigas MA, Eng C, Feig BW, Das P, Krishnan S, Crane CH, $\mathrm{Hu} \mathrm{CY}$, Chang GJ. Neoadjuvant treatment responseas an early response indicator for patients with rectal cancer. J Clin Oncol. 2012; 30:1770-6.

7. Kaplan E MP. Nonparametric estimation from incomplete observations. J Am Stat Assoc. 1958; 53:457-481.

8. Mantel N. Evaluation of survival data and two new rank order statistics arising in its consideration. Cancer Chemother Rep. 1966; 50:163-170.

9. Gill RD. Multistate life-tables and regression models. Math Popul Stud. 1992; 3:259-276.

10. Oh SY, Bae Kim Y, Wook Suh K. Oncologic Outcome of ypT1-2N0 Rectal Cancer After Neoadjuvant
Chemoradiotherapy Compared With pT1-2N0 Rectal Cancer. Am J Clin Oncol. 2015.

11. Du CZ, Chen YC, Cai Y, Xue WC, Gu J. Oncologic outcomes of primary and post-irradiated early stage rectal cancer: a retrospective cohort study. World J Gastroenterol. 2011; 17:3229-34.

12. Chen J, Lu H, Yan D, Cui F, Wang X, Yu F, Xue Y, Feng X, Wang J, Wang X, Jiang T, Zhang M, Zhao S, Yu Y, Tang H, Peng Z. PAK increase chemoresistance and is a prognostic marker for stage II and III colon cancer patients undergoing 5-FU based chemotherapy. Oncotarget. 2015; 6:355-67.

13. Kuebler JP, Wieand HS, O'Connell MJ, Smith RE, Colangelo LH, Yothers G, Petrelli NJ, Findlay MP, Seay TE, Atkins JN, Zapas JL, Goodwin JW, Fehrenbacher L Ramanathan, RK Conley, BA Flynn, PJ et al. Oxaliplatin combined with weekly bolus fluorouracil and leucovorin as surgical adjuvant chemotherapyfor stage II and III colon cancer: results from NSABP C-07. J Clin Oncol. 2007; 25:2198-204.

14. André $\mathrm{T}$, Boni $\mathrm{C}$, Navarro $\mathrm{M}$, Tabernero J, Hickish $\mathrm{T}$, Topham C, Bonetti A, Clingan P, Bridgewater J, Rivera F, de Gramont A. Improved overall survival with oxaliplatin, fluorouracil, and leucovorin as adjuvant treatment in stage II or III colon cancer in the MOSAIC trial. J Clin Oncol. 2009; 27:3109-16.

15. Haller DG, Tabernero J, Maroun J, de Braud F, Price T, Van Cutsem E, Hill M, Gilberg F, Rittweger K, Schmoll HJ. Capecitabine plus oxaliplatin compared with fluorouracil and folinic acid as adjuvant therapy for stage III colon cancer. J Clin Oncol. 2011; 29:1465-71.

16. Petersen SH, Harling H, Kirkeby LT, Wille-Jørgensen P, Mocellin S. Postoperative adjuvant chemotherapy in rectal cancer operated for cure. Cochrane Database Syst Rev. 2012; 3:CD004078.

17. Bosset JF, Collette L, Calais G, Mineur L, Maingon P, Radosevic-Jelic L, Daban A, Bardet E, Beny A, Ollier JC. Chemotherapy with preoperative radiotherapy in rectal cancer. N Engl J Med. 2006; 355:1114-23.

18. Collette L, Bosset JF, den Dulk M, Nguyen F, Mineur L, Maingon P, Radosevic-Jelic L Piérart, M Calais. Patients with curative resection of cT3-4 rectal cancer after preoperative radiotherapy or radiochemotherapy: does anybody benefit from adjuvant fluorouracil-based chemotherapy? A trial of the European Organisation for Research and Treatment of Cancer Radiation Oncology Group. J Clin Oncol. 2007; 25:4379-86.

19. Fietkau R, Barten M, Klautke G, Klar E, Ludwig K, Thomas H, Brinckmann W, Friedrich A, Prall F, Hartung G, Küchenmeister U, Kundt G. Postoperative chemotherapy may not be necessary for patients with ypN0-category after neoadjuvant chemoradiotherapy of rectal cancer. Dis Colon Rectum. 2006; 49:1284-92.

20. Gao P, Song YX, Sun JX, Chen XW, Xu YY, Zhao JH, Huang XZ, Xu HM, Wang ZN. Which is the best postoperative chemotherapy regimen in patients with rectal cancer after neoadjuvant therapy? BMC Cancer. 2014; 14:888. 